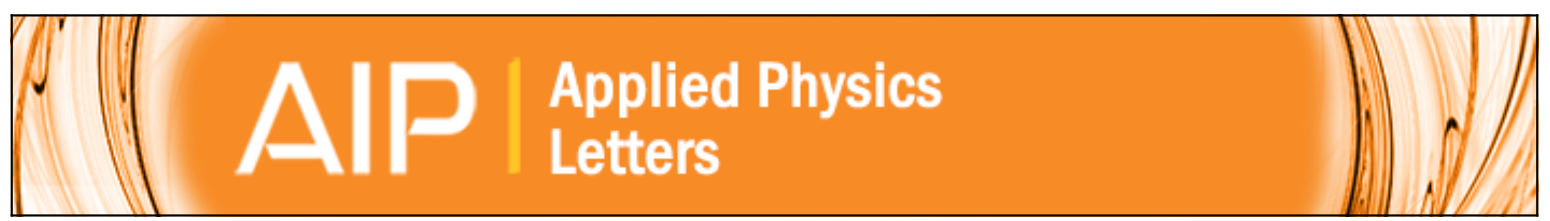

\title{
Integrated nanostructures for direct detection of DNA at attomolar concentrations
}

Leyla Soleymani, Zhichao Fang, Shana O. Kelley, and Edward H. Sargent

Citation: Applied Physics Letters 95, 143701 (2009); doi: 10.1063/1.3226103

View online: http://dx.doi.org/10.1063/1.3226103

View Table of Contents: http://scitation.aip.org/content/aip/journal/apl/95/14?ver=pdfcov

Published by the AIP Publishing

\section{Articles you may be interested in}

Biofunctionalized AIGaN/GaN high electron mobility transistor for DNA hybridization detection

Appl. Phys. Lett. 100, 232109 (2012); 10.1063/1.4727895

Gold nanoring as a sensitive plasmonic biosensor for on-chip DNA detection

Appl. Phys. Lett. 100, 173114 (2012); 10.1063/1.4707382

Selective placement of DNA origami on substrates patterned by nanoimprint lithography

J. Vac. Sci. Technol. B 29, 06F205 (2011); 10.1116/1.3646900

Focusing and trapping of DNA molecules by head-on ac electrokinetic streaming through join asymmetric polarization

Biomicrofluidics 4, 034108 (2010); 10.1063/1.3481468

A paralleled readout system for an electrical DNA-hybridization assay based on a microstructured electrode array

Rev. Sci. Instrum. 74, 1077 (2003); 10.1063/1.1533103

\section{AIP $\left.\right|_{\text {APL Photonics }}$}

APL Photonics is pleased to announce Benjamin Eggleton as its Editor-in-Chief

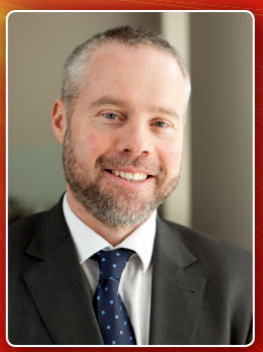




\title{
Integrated nanostructures for direct detection of DNA at attomolar concentrations
}

\author{
Leyla Soleymani, ${ }^{1}$ Zhichao Fang, ${ }^{2}$ Shana O. Kelley, ${ }^{2,3, a)}$ and Edward H. Sargent ${ }^{1, b)}$ \\ ${ }^{1}$ Department of Electrical and Computer Engineering, Faculty of Engineering, University of Toronto, \\ Toronto, Ontario M5S 3G4, Canada \\ ${ }^{2}$ Department of Pharmaceutical Sciences, Leslie Dan Faculty of Pharmacy, University of Toronto, \\ Toronto, Ontario M5S 3M2, Canada \\ ${ }^{3}$ Department of Biochemistry, Faculty of Medicine, University of Toronto, Toronto, \\ Ontario M5S 1A8, Canada
}

(Received 17 June 2009; accepted 17 August 2009; published online 5 October 2009)

\begin{abstract}
We report an integrated chip that senses nucleic acid biomarkers at exceptionally low concentrations. To achieve such sensitivities we exploit four concepts. (1) Nanostructured electrodes allow efficient display of probe sequences. (2) The use of uncharged probe sequences lowers the background signal in our read-out system. (3) Electrocatalysis provides built-in amplification of the electrical signal that reports hybridization events. (4) An optimal self-assembled monolayer of thiol-functionalized probe molecules is best achieved with the aid of a short spacer molecule to confer enhanced accessibility. We show herein that via joint optimization along these four axes we achieve attomolar sensitivity. (c) 2009 American Institute of Physics. [doi:10.1063/1.3226103]
\end{abstract}

Sensitive and specific detection of biomarkers is of critical importance in clinical diagnostics as well as in biological research. ${ }^{1}$ Nucleic acids are biomarkers of central importance, bringing a great deal of attention to the development of nucleic acids biosensors. ${ }^{2}$ In previous reports, the surface of a sensor is typically modified with nucleic acids probes capable of generating an optical, ${ }^{3-8}$ gravimetric, ${ }^{9}$ piezoelectric, ${ }^{10}$ electrical or electrochemical ${ }^{11-16}$ signal when hybridization with a complementary sequence occurs.

Clinical use of direct nucleic acids detection-sensing the presence of complementary strands without resorting to enzymatic amplification methods such as Polymerase Chain Reaction (PCR) - demands further progress in sensitivity as well as specificity. Electrochemical detection of DNA hybridization has been realized in several systems, ${ }^{17,18}$ and in principle offers the capability to detect small number of analyte molecules in solution with the aid of very simple, low-cost instrumentation. Previous studies have reported detection limits as low as 80 molecules based on multistep enzymatic labeling. ${ }^{19}$ Advances in specificity ${ }^{20}$ have allowed the identification of single base-pair mismatches. Nonetheless, sensitivity, specificity, and the capacity to multiplex a number of biomarkers on a single chip are crucial features that have yet to be combined in a single system.

Here we report that a chip-based array of nanotextured microelectrodes allows ultrasensitive, label-free discrimination of complementary, and noncomplementary DNA analytes. We tether capture probes made of peptide nucleic acid $(\mathrm{PNA})^{21}$ to our electrodes. When the analyte solution is introduced, only complementary targets hybridize with our surface-anchored probe molecules. The resultant capture of negatively charged DNA molecules is transduced as a readily measured electrical current using the electrocatalytic scheme $^{22}$ developed in our laboratories. In this approach, electrochemical current is generated by reduction of posi-

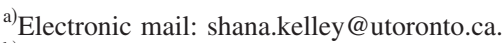

b) Electronic mail: ted.sargent@utoronto.ca.
}

tively charged $\mathrm{Ru}\left(\mathrm{NH}_{3}\right)_{6}^{3+}$, a DNA-binding electron acceptor. The electrical current is further amplified by introducing $\mathrm{Fe}(\mathrm{CN})_{6}^{3-}$ that serves as an anionic electron acceptor. The result is an electrocatalytic reporter system for high-gain hybridization-event signal transduction.

Here we describe the systematic optimization of this system resulting in an unparalleled combination of sensitivity, specificity, and integration. We begin with a highly nanostructured electrode and prove that sensitivity is vastly enhanced when a conventional thiolated DNA probe (a charged molecule) is replaced with a neutral PNA probe. Moreover, we find that only by depositing a mixed monolayer-one that contains both the capture probes and also short, uncharged molecules used to moderate the probe density and block the sensor surface from nonspecific adsorption of mismatched DNA targets-can we reach below the femtomolar detection limit. With these optimizations, our detection system allows us to detect fewer than 100 target DNA molecules.

To create a platform for a multiplexed chip, we use conventional photolithographic fabrication to build our chip as a template for ensuing controlled deposition of nanomaterials in an individually electrically addressed array. Figure 1(a) shows the schematic of the passive electronic chip. A $350 \mathrm{~nm}$ thick layer of gold is evaporated and subsequently photolithographically patterned on the $\mathrm{Si} / \mathrm{SiO}_{2}$ substrate to provide for later connection of the functionalized nanostructures that will reside at the tips of $5 \mu \mathrm{m}$ wide planar Au wires to millimeter-sized bond pads for connection to off-chip instrumentation. The entire chip is then covered with a pinholefree layer of insulating $\mathrm{SiO}_{2} .500 \mathrm{~nm}$ circular apertures are dry etched to expose the underlying gold layer. This step in the chip fabrication defines the template for the precise location of the subsequently grown nanostructured sensing elements.

Motivated by previous reports showing that nanostructuring can provide major improvements in the detection limits of biosensors, ${ }^{23-25}$ we sought to incorporate such nanostructures on our microelectrodes. We found that elec- 
(a)

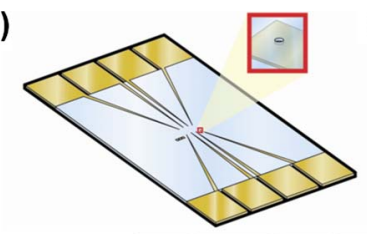

(b)

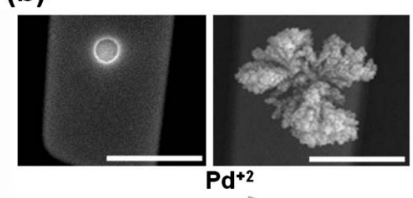

(C)

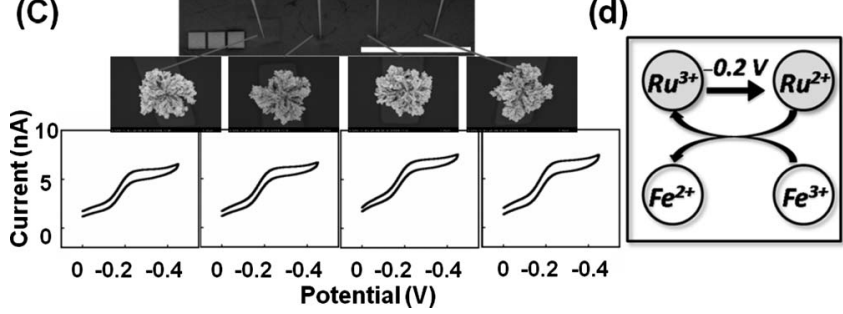

FIG. 1. (Color online) (a) Schematic representation of the photolithographically defined chip (b) Scanning electron microscopy (SEM) image showing aperture before (left) and after (right) template-directed electrodeposition. Scale bar represents $4 \mu \mathrm{m}$. (c) SEM image (top) and the electrochemical response (bottom) of four electrodes on the same chip. (d) Schematic illustrating the electrocatalytic reporter system.

trodeposition of $\mathrm{Au}, \mathrm{Pd}$, and $\mathrm{Pt}$ resulted in nanostructured microelectrodes when a suitable plating potential and supporting electrolyte was chosen. However, an extensive analysis revealed that Pd provided the best performance when modified with thiolated capture probes. This is consistent with prior work demonstrating stronger interaction of $\mathrm{Pd}$ with thiols compared with $\mathrm{Au}$ and $\mathrm{Pt}^{26}{ }^{26}$ Following these findings, we used electrodeposition through the lithographically defined apertures to reduce palladium ions in solution in order to produce metallic nanostructures, filling the apertures with metal structures that fan out above the top surface of the oxide as shown in the SEM images in Figs. 1(b) and 1(c). We investigate the robustness and reproducibility of these electrodes in Fig. 1(c): cyclic voltammetry (CV) scans, generated using a three-electrode system in which nanotextured microelectrodes (NMEs) serving as working electrodes immersed in $3 \mathrm{mM} \mathrm{Ru}\left(\mathrm{NH}_{3}\right)_{6}^{3+}$, show ideal microelectrode behavior. ${ }^{27}$ Lead-to-lead CV variation is less than 5\% among nominally identically grown NMEs.

With a view of creating a DNA sensor having high sensitivity and specificity, we designed and compared different probe structures: one made of conventional DNA with its phosphodiester backbone, the other employing PNA having a pseudopeptide backbone. Both of these probes are known to hybridize with complementary DNA strands according to Watson-Crick hydrogen-bonding rules; however, unlike DNA, PNA is a charge-neutral molecule. As a result, its electrocatalytic background signal in case of an unhybridized probe monolayer is expected to be dramatically lower than that of DNA. ${ }^{28}$

To capture specific analytes in solution, single-stranded DNA and PNA strands terminated with a single thiol endgroup (as described elsewhere ${ }^{29}$ ) were immobilized on the electrodes. To evaluate further the hybridization of the electrode-bound probe strands with target analyte strands, the electrodes (DNA- or PNA-modified) were incubated with $10 \mathrm{pM}$ complementary target for $1 \mathrm{~h}$ at $37^{\circ} \mathrm{C}$. The electrodes were then removed from the analyte solution, rinsed, and immersed in an electrocatalytic solution depicted in
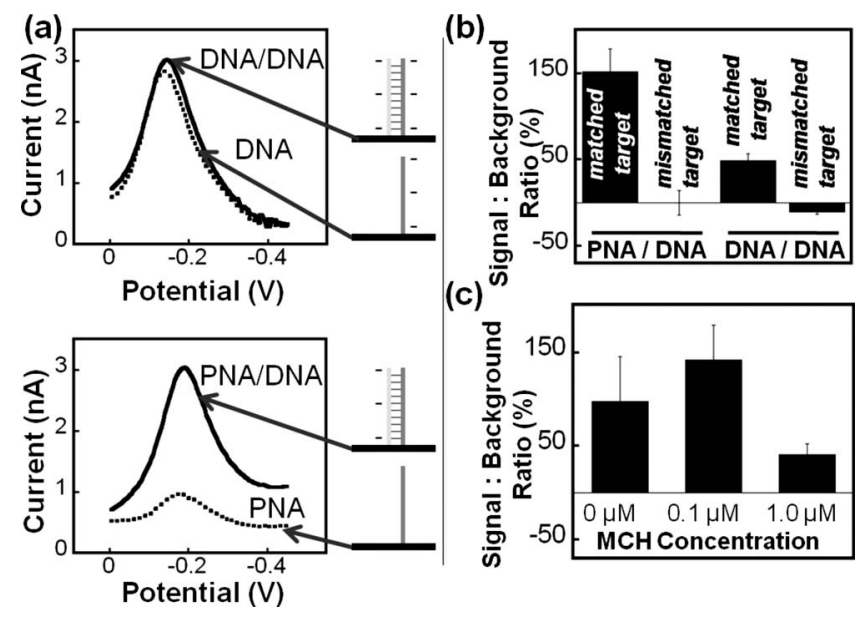

FIG. 2. (a) Schematic illustration and differential pulse voltammetry measurements representing hybridization with a DNA probe (top) versus a PNA probe (bottom). (b) Signal to background ratio comparison of a DNA modified device and a PNA modified device. (c) Signal to background ratio comparison of PNA devices having different $\mathrm{MCH}$ spacer concentrations.

Fig. 1(d) to enable the read-out of the state of hybridization of its functionalized surface.

We show in Fig. 2(a) differential pulse voltammetry traces for the DNA-versus PNA-functionalized chips. PNAmodified electrodes show a 70\% lower background current compared to DNA-modified electrodes. As expected, the PNA-only electrode produces little electrocatalytic signal.

As a consequence of elevated signal and lowered background, the use of PNA produces a dramatic threefold enhancement in signal-to-background ratio compared to DNA probe in analyzing $10 \mathrm{pM}$ complementary target. The use of the uncharged probe layer thus enhances capture of the negatively charged analyte molecules, providing a further advantage to the use of PNA.

We carried out a suite of control experiments that would challenge the specificity of the system and reveal any artifacts arising due to nonspecific adsorption of target molecules from the analyte solution. When we introduced mismatched target at an elevated concentration of $100 \mathrm{pM}$, the signal change [Fig. 2(b)] under these nonspecific conditions remains well below 14 percent.

Electrode nanostructuring, electrocatalysis, and PNA electrode modification made possible the detection of $1 \mathrm{fM}$ of analyte; this detection limit requires having thousands of DNA molecules in solution. Clinically relevant, amplification-free nucleic acids detection to aid in disease diagnosis requires detection of hundreds of molecules, pointing to the need for further optimization.

To decrease the extent of nonspecific adsorption on the electrode surface and to find a more favorable density of capture probes, ${ }^{30,31}$ we endeavored to functionalize the system using a mixed-monolayer of probe and spacer/blocker molecules. We thus added to our use of PNA probe a mercaptohexanol (MCH) blocker/spacer, a short, uncharged molecule. We believed that an optimal MCH concentration would exist: its concentration high enough to favor specific hybridization of complementary DNA over nonspecific adsorption while allowing a sufficient PNA probe density to capture analyte DNA and produce detectable signal changes.

As shown in Fig. 2(c), $0.1 \mu \mathrm{M} \mathrm{MCH}$ produced a 300\% largen signal change than $1, \mu \mathrm{M}$ MCH and $50 \%$ enhance- 
(a)

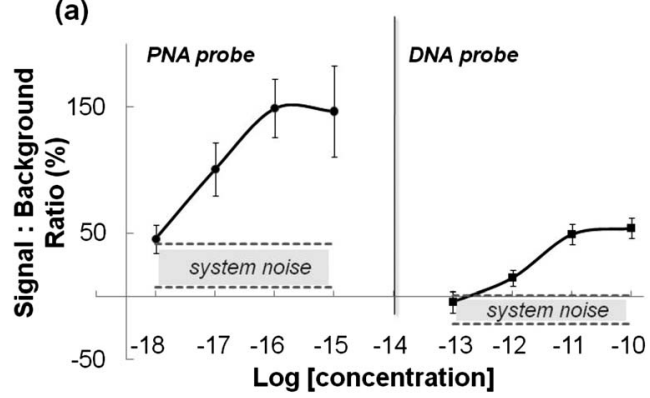

(b) Log [concentration]

Complementary target: ${ }^{5}$ AGC GCG GCA GGA AGC CTT AT ${ }^{3}$ Non-complementary target: ${ }^{\prime}$ 'TTT TTT TTT TTT TTT TTT TT ${ }^{3 '}$ DNA probe: SH-5'ATA AGG CTT CCT GCC GCG CT ${ }^{3}$ ' PNA probe: $\mathrm{NH}_{2}$-Cys-Gly-ATA AGG CTT CCT GCC GCG CT-CONH

FIG. 3. (a) Signal to background ratio of a PNA device (left) and a DNA device (right) at different concentrations of complementary target. The system noise associated with hybridization with noncomplementary target is shown for reference. The devices contained $0.1 \mu \mathrm{M} \mathrm{MCH}$ acting as a spacer. (b) Sequences of the nucleic acid targets and probes used herein.

ment relative to the case in which no $\mathrm{MCH}$ was employed. The use of this spacer/blocker layer thus proved a powerful additional implement in the quest for exceptional sensitivity.

The ultimate goal of this sensing platform is the analysis of clinical samples containing a small number of copies of a particular strand within the large background present in a heterogeneous sample. We critically evaluated the specific detection limit of our PNA-featured approach by analyzing $10 \mu \mathrm{L}$ solutions containing between $1 \mathrm{aM}$ and $1 \mathrm{fM}$ complementary target alongside solutions containing $100 \mathrm{fM}$ noncomplementary targets. To demonstrate further the advantages presented by the PNA probe we evaluated the limit of detection of the DNA probe device by looking between 100 $\mathrm{fM}$ and $100 \mathrm{pM}$ side by side of $100 \mathrm{pM}$ noncomplementary targets. As shown in Fig. 3(a), we obtain a $10 \mathrm{aM}$ limit of detection using our optimized chip-based platform as opposed to $10 \mathrm{pM}$ detection limit realized with our DNA device. [Target and probe sequences are summarized in Fig. 3(b).] This six orders of magnitude enhancement in sensitivity corresponds to the specific detection of fewer than 100 copies of the target sequence.

The system reported herein offers the low cost and convenience of simple photolithographic fabrication, yet combines it with the power of nanostructuring to elevate biomolecular detection sensitivity. It provides label-free ultrasensitive biomarker detection through the joint optimization of the use of uncharged PNA probes, an electrocatalytic reporter for electronic signal amplification, and a molecular spacer that selects in favor of specific hybridization over nonspecific adsorption.
The authors gratefully acknowledge the financial support from the Ontario Centres of Excellence, Genome Canada, the Ontario Ministry of Research and Innovation, and NSERC 484838.

${ }^{1}$ F. W. Scheller, U. Wollenberger, A. Warsinke, and F. Lisdat, Curr. Opin. Biotechnol. 12, 35 (2001).

${ }^{2}$ M. Baker, Nat. Biotechnol. 24, 931 (2006).

${ }^{3}$ G. Rong, J. D. Ryckman, R. L. Mernaugh, and S. M. Weiss, Appl. Phys. Lett. 93, 161109 (2008).

${ }^{4}$ X. Wang, K. L. Cooper, A. Wang, J. Xu, Z. Wang, Y. Zhang, and Z. Tu, Appl. Phys. Lett. 89, 163901 (2006).

${ }^{5}$ R. Jin, Y. C. Cao, C. S. Thaxton, and C. A. Mirkin, Small 2, 375 (2006).

${ }^{6}$ L. Fabris, M. Dante, G. Braun, S. Joon Lee, N. O. Reich, M. Moskovits, T. Nguyen, and G. C. Bazan, J. Am. Chem. Soc. 129, 6086 (2007).

${ }^{7}$ X. Li, J. Qian, L. Jiang, and S. He, Appl. Phys. Lett. 94, 063111 (2009).

${ }^{8}$ T. T. Goodrich, H. J. Lee, and R. M. Corn, J. Am. Chem. Soc. 126, 4086 (2004)

${ }^{9}$ I. Mannelli, M. Minunni, S. Tombelli, R. Wang, M. M. Spiriti, and M. Mascini, Bioelectrochemistry 66, 129 (2005).

${ }^{10}$ M. Su, S. Li, and V. P. Dravid, Appl. Phys. Lett. 82, 3562 (2003).

${ }^{11}$ C. Chen, F. Ko, C. Chen, T. Liu, E. Y. Chang, Y. Yang, S. Yan, and T. Chu, Appl. Phys. Lett. 91, 253103 (2007).

${ }^{12}$ B. M. Zeglis and J. K. Barton, Nat. Protoc. 2, 357 (2007).

${ }^{13}$ M. Lapierre-Devlin, C. L. Asher, B. J. Taft, R. Gasparac, M. A. Roberts, and S. O. Kelley, Nano Lett. 5, 1051 (2005).

${ }^{14}$ S. Park, T. A. Taton, and C. A. Mirkin, Science 295, 1503 (2002).

${ }^{15}$ J. Hahm and C. M. Lieber, Nano Lett. 4, 51 (2004).

${ }^{16}$ H. Lee, J. Park, J. Kim, H. Jung, and T. Kawai, Appl. Phys. Lett. 89, 113901 (2006).

${ }^{17}$ H. Lord and S. O. Kelley, J. Mater. Chem. 19, 3127 (2009).

${ }^{18}$ T. G. Drummond, M. G. Hill, and J. K. Barton, Nat. Biotechnol. 21, 1192 (2003).

${ }^{19}$ B. Munge, G. Liu, G. Collins, and J. Wang, Anal. Chem. 77, 4662 (2005)

${ }^{20}$ K. Kerman, M. Saito, Y. Morita, Y. Takamura, M. Ozsoz, and E. Tamiya, Anal. Chem. 76, 1877 (2004).

${ }^{21}$ M. Egholm, O. Buchardt, L. Christensen, C. Behrens, S. M. Freier, D. A. Driver, R. H. Berg, S. K. Kim, B. Norden, and P. E. Nielsen, Nature (London) 365, 566 (1993).

${ }^{22}$ M. A. Lapierre, M. O'Keefe, B. J. Taft, and S. O. Kelley, Anal. Chem. 75, 6327 (2003)

${ }^{23}$ V. Mani, B. V. Chikkaveeraiah, V. Patel, J. S. Gutkind, and J. F. Rusling, ACS Nano 3, 585 (2009).

${ }^{24}$ L. Soleymani, Z. Fang, X. Sun, H. Yang, B. J. Taft, E. H. Sargent, and S. O. Kelley, "Nanostructuring of patterned microelectrodes to enhance the sensitivity of electrochemical nucleic acids detection," Angew. Chem. (in press).

${ }^{25}$ L. Soleymani, Z. Fang, E. H. Sargent, and S.O. Kelley, "Programming the sensitivity of biosensors through controlled nanostructuring of electrodes," Nat. Nanotechnol.

${ }^{26}$ J. C. Love, D. B. Wolfe, R. Haasch, M. L. Chabinyc, K. E. Paul, G. M. Whitesides, and R. G. Nuzzo, J. Am. Chem. Soc. 125, 2597 (2003).

${ }^{27}$ J. Heinze, Angew. Chem., Int. Ed. Engl. 32, 1268 (1993).

${ }^{28}$ Z. Fang and S. O. Kelley, Anal. Chem. 81, 612 (2009).

${ }^{29}$ B. J. Taft, M. O'Keefe, J. T. Fourkas, and S. O. Kelley, Anal. Chim. Acta 496, 81 (2003).

${ }^{30}$ A. K. R. Lytton-Jean and C. A. Mirkin, J. Am. Chem. Soc. 127, 12754 (2005).

${ }^{31}$ T. M. Herne and M. J. Tarlov, J. Am. Chem. Soc. 119, 8916 (1997). 